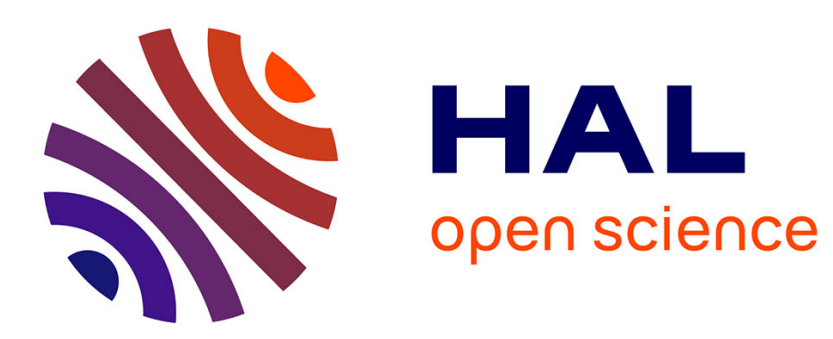

\title{
EVOLUTION THERMIQUE DES GELS D'ALUMINO SILICATE ALCALIN
}

T. Woignier, J. Phalippou, J. Zarzycki

\section{To cite this version:}

T. Woignier, J. Phalippou, J. Zarzycki. EVOLUTION THERMIQUE DES GELS D'ALUMINO SILICATE ALCALIN. Journal de Physique Colloques, 1982, 43 (C9), pp.C9-261-C9-264. 10.1051/jphyscol:1982948 . jpa-00222477

\section{HAL Id: jpa-00222477 https://hal.science/jpa-00222477}

Submitted on 1 Jan 1982

HAL is a multi-disciplinary open access archive for the deposit and dissemination of scientific research documents, whether they are published or not. The documents may come from teaching and research institutions in France or abroad, or from public or private research centers.
L'archive ouverte pluridisciplinaire HAL, est destinée au dépôt et à la diffusion de documents scientifiques de niveau recherche, publiés ou non, émanant des établissements d'enseignement et de recherche français ou étrangers, des laboratoires publics ou privés. 
JOURNAL DE PHYSIQUE

Colloque C8, supplément au no12, Tome 43, décembre 1982

page C9-261

\title{
EVOLUTION THERMIQUE DES GELS D'ALUMINO SILICATE ALCALIN
}

\author{
T. Woignier, J. Phalippou et J. Zarzycki \\ Laboratoire des Sciences des Matériaux et Laboratoire des Verres du CNRS, Ini- \\ versité de Montpellier. II, Place Eugène Batailzon, 34060 Montpellier Cedex, France
}

Résumé. Les gels de composition $\mathrm{K}_{2} \mathrm{O}-\mathrm{Al}_{2} \mathrm{O}_{3}-6 \mathrm{SiO}_{2}, \mathrm{Na}_{2} \mathrm{O}-\mathrm{Al}_{2} \mathrm{O}_{3}-6 \mathrm{SiO}_{2}$, Li $\mathrm{L}_{2} \mathrm{O}-$ $\mathrm{Al}_{2} \mathrm{O}_{3}-6 \mathrm{SiO}_{2}$ ont été préparés suivant deux techñiques différentes ${ }^{2}$ : par destabilisation d'une solution colloidale de silice et par hydrolyse et polycondensation du Si( $\left.\mathrm{OCH}_{3}\right)_{4} . \mathrm{Al}_{2} \mathrm{O}_{3}, \mathrm{Na}_{2} \mathrm{O}, \mathrm{K}_{2} \mathrm{O}, \mathrm{Li} \mathrm{O}_{2} \mathrm{O}$ ont été rajoutés sous forme de nitrates. La cristallisation de ces gêls a été étudiée en fonction de la température par diffraction des Rayons X. Si tous les gels présentent à faible température des bandes IR et des pics RX caractéristiques des nitrates, ces derniers se décomposent à plus haute température. La structure devient alors amorphe. Pour des températures plus élevées les gels contenant du potassium présentent la plus faible tendance à la cristallisation. Le retrait du gel est important à basse température il a été suivi par dilatométrie.

\begin{abstract}
The gels of composition $\mathrm{K}_{2} \mathrm{O}-\mathrm{AI}_{2} \mathrm{O}_{3}-6 \mathrm{SiO}_{2}, \mathrm{Na}_{2} \mathrm{O}-\mathrm{Al}_{2} \mathrm{O}_{3}-6 \mathrm{SiO}_{2}$, $\mathrm{Li} \mathrm{i}_{2} \mathrm{O}-$ $\overline{\mathrm{Al}} \mathrm{O}_{3}-6 \mathrm{SiO}_{2}$ were prepared following two different techniques, destabilization of a colloidal solution of silica and hydrolysis and polycondensation of $\mathrm{Si}\left(\mathrm{OCH}_{3}\right)_{4} \cdot \mathrm{AI}_{2} \mathrm{O}_{3}, \mathrm{Na}, \mathrm{O}, \mathrm{K}_{2} \mathrm{O}$, Ii $\mathrm{i}_{2} \mathrm{O}$ were added in the form of nitrates in aqueous solution. The crystaliization of these gels was studied as a function of temperature by X-Ray diffraction. At low temperature all these gels show characteristic $X$ Ray peaks and also I.R. bands due to the presence of different nitrates. These nitrates however decompose at higher temperatures; consequently, the structure becomes amorphous. At more elevated temperature the gel containing $\mathrm{KNO}_{3}$ shows a weaker tendency to crystallize. The contraction of the gel was found to be important at low temperatures as observed by dilatometry measurements.
\end{abstract}

1. Introduction. - Les gels et les verres ont pour trait commun leur caractère amorphe. Les gels se différencient des verres par le fait qu'ils possèdent une grande surface spécifique, une porosité élevée et renferment généralement une forte teneur en groupements hydroxyles. Théoriquement il suffit de chauffer un gel pour que ces différences disparaissent. En fait, hormis quelques cas trés particuliers de systèmes réfractaires (1) cela n'est pas aussi simple. Le gel lors du chauffage bien souvent cristallise. Il est bien connu que certains verres sont difficilement dévitrifiables ; c'est le cas de l'orthoclase $\left(\mathrm{K}_{2} \mathrm{O}-\mathrm{Al}_{2} \mathrm{O}_{3}-6 \mathrm{SiO}_{2}\right)$. De plus l'alumine est souvent rajoutée a des compositions verrières pour üiminuêr la tendance à la cristallisation. Le but de notre travail était de savoir si lescritèresqui ont été énoncés pour rendre compte de la plus ou moins grande résistance à la cristallisation des verres le sont aussi pour les gels. Pour cela trois compositions ont été élaborées de telle sorte que la teneur en silice et en alumine restait constante. Seule la nature de l'alcalin a été modifiée. Nous avons porté notre choix sur des compositions pour lesquelles les quantités d'oxyde alcalin et d'alumine sont égales; ce qui nous assurait d'avoir un maximum d'atomes d'oxygènes pontants (2).

2. Procédures expérimentales : Corrélativement avec l'action des alcalins sur la cristallisation, il était intéressant de comparer les comportenents des gels élaborés par deux méthodes depuis longtemps utilisées au laboratoire (3). La première consiste en une destabilisation d'une solution colloldale de silice commercialisée sous le nom de ludox AS 40 (gel 1) la deuxième esț basée sur une réaction d'hydrolyse et de polycondensation du tetramethoxysilane en milieu methanolique (gel 2). 
Les ions alcalins et l'aluminium ont été introduits sous forme de nitrates en solution. Les solutions minérales et organiques des composés du silicium ont été préparées à $\mathrm{P} h=2,5$ par ajout $\mathrm{d}^{\prime} \mathrm{HNO}_{3}$, afin d'éviter la précipitation de l'hydroxyde d'aluminium. Pour les gels 2 les volumes de $\mathrm{CH}_{3} \mathrm{OH}$ et de $\left.\mathrm{Si}_{(\mathrm{OCH}}\right)_{4}$ étaient identiques. Aprés trois semaines de vieillissement dans des tubes hermétiques, I'exposition du gel à I'aix permettait le départ du solvant. Les traitements thermiques ont été effectués aprés une semaine de séchage à température ambiante complété par $24 h$ dans une étuve à $60^{\circ} \mathrm{C}$.

L'évolution structurale a été suivie par diffraction des rayons $X$.

3. Résultats : tous les traitements thermiques ont une durée d'une heure.

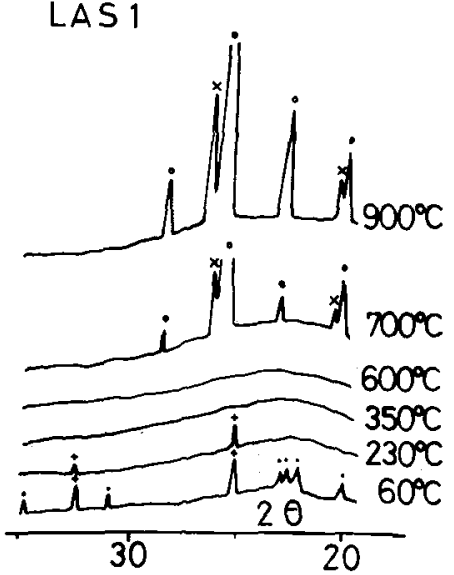

Fig. 1

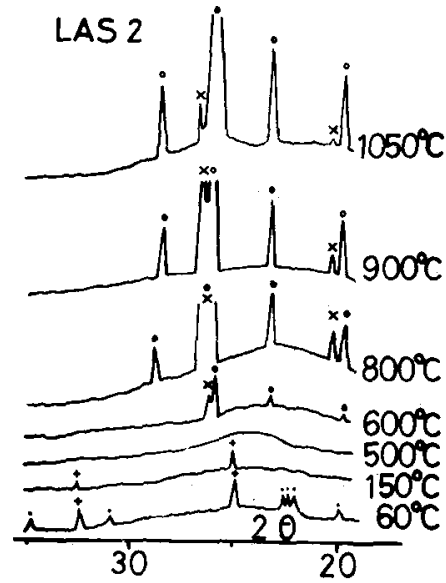

Fig. 2

a) Composition $\mathrm{Li}, \mathrm{O}_{2}-\mathrm{Al}_{2} \mathrm{O}_{3}-6 \mathrm{SiO}_{2}$ (LAS). Les figures 1 et 2 montrent 1 'ëvolution des diffractogrammes en fonction de la température des gels LAS 1 et LAS 2.

Dès $60^{\circ} \mathrm{C}$ il y a présence de phases cristallines : nitrate d'aluminium hydraté, $\mathrm{A}_{1 \mathrm{NO}} \mathrm{H}_{3}$ $9 \mathrm{H}_{2} \mathrm{O}_{1}$ monoclinique (.) et nitrate de lithium, LiNO 3 , trigonal $(+)$.

Par la suite le nitrate d'aluminium disparait entre $60^{\circ} \mathrm{C}$ et $150^{\circ} \mathrm{C}$, le départ du nitrate de lithium s'effectue entre $230^{\circ} \mathrm{C}$ et $350^{\circ} \mathrm{C}$ pour le gel LAS 1 et entre $130^{\circ} \mathrm{C}$ et $230^{\circ} \mathrm{C}$ pour le gel LAS 2. A plus haute tempêrature le gel devient amorphe.

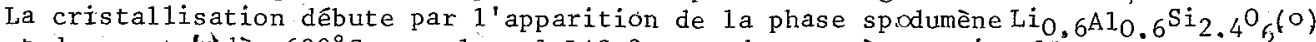
et de quartz(x)dès $600^{\circ} \mathrm{C}$ pour lè gel LAS 2 ; ces deux espèces cristalines sont présentes à $700^{\circ} \mathrm{C}$ pour le gel 1 .

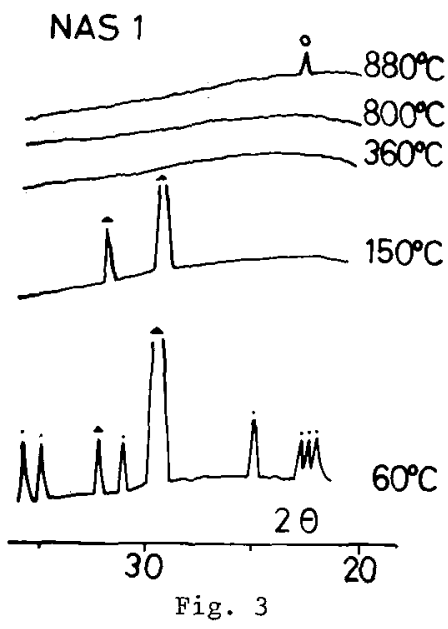

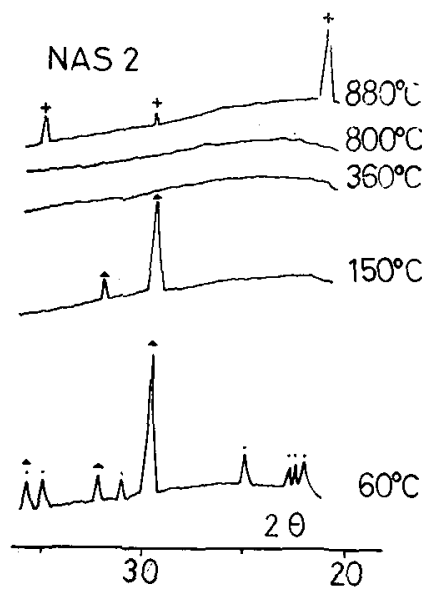

Fig. 4 
b) composition $\mathrm{Na}_{2} \mathrm{O}-\mathrm{Al}_{2} \mathrm{O}_{3}-6 \mathrm{SiO}_{2}$ (NAS). Sur les figures 3 et 4 nous pouvons suivre le comportement thermique des gels NASl et NAS2. De la même façon que pour le gel de lithium nous observons à $60^{\circ} \mathrm{C}$ la présence de nitrates cristallins ; $\mathrm{AlNO}_{3}, 9 \mathrm{H}_{2} \mathrm{O}$ (.) ainsi que le nitrate de sodium $\mathrm{NaNO}_{3}$ hexagonal ( $\mathbf{\Lambda}$. La décomposition de $\mathrm{AlNO}_{3}, 9 \mathrm{H}_{2} \mathrm{O}$ s'effectúe entre. 60 et $150^{\circ} \mathrm{C}$, celle de $\mathrm{NaNO}_{3}$ entre $150^{\circ} \mathrm{C}$ et $260^{\circ} \mathrm{C}$. La cristallisat: tion s'amorce vers $380^{\circ} \mathrm{C}$; i.1 s'agit du composé $\mathrm{Na}$ Al SiO4(+) pour le gel 2 et de 1 'albite NaAlsi ${ }_{3} \mathrm{O}_{8}$ (0) pour le gel 1 .
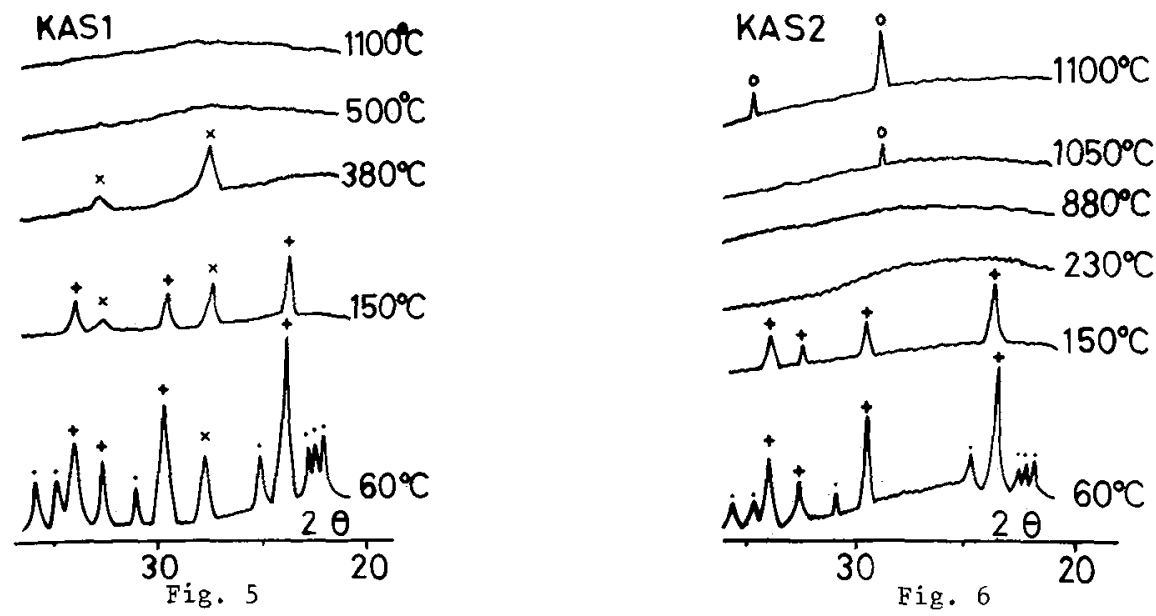

c) composition $\mathrm{K}_{2} \mathrm{O}-\mathrm{Al}_{2} \mathrm{O}_{3}-6 \mathrm{SiO}_{2}$ (KAS). Comme dans les deux cas précédents $\mathrm{AINO}_{3}, 9 \mathrm{H}_{2}-$ se décompose entre $60^{\circ} \mathrm{C}$ et $150^{\circ} \mathrm{C}$. Par la suite il y a disparition des phases nitra tes de potassium $(t, x) \cdot \mathrm{KNO}_{3}$ orthorhombique $(+) \mathrm{n}^{\prime}$ est plus présent à $230^{\circ} \mathrm{C}$; la phase hexagonale $(x)$ qui n'existait que dans le cas du gel 1 disparait entre $400^{\circ} \mathrm{C}$ et $500^{\circ} \mathrm{C}$. Au delà, le gel 1 reste amorphe même à $1100^{\circ} \mathrm{C}$; par contre Kasz présente une faible cristallisation, dès $1050^{\circ} \mathrm{C}$, de Kaliophylite hexagonale KAlSiO 4 (o).

Lues essais de mesure de retrait par dilatométrie différentielle mettent en évidence le manque de tenue mécanique des matériaux. Les vitesses de frittage mesurées à basse température sont importantes. Les discontinuités observées sur les graphes correspondent au départ des nitrates $.85^{\circ} \mathrm{C}$ pour $\mathrm{AlNO}_{3}, 9 \mathrm{H}_{2} \mathrm{O}$, $205^{\circ} \mathrm{C}$ pour $\mathrm{LiNO}_{3}$ et $175^{\circ} \mathrm{C}$ pour $\mathrm{NaNO}_{3}$. Les échantillons traités à $60^{\circ} \mathrm{C}$ présentent une faible dilatation dûe vraisemblablement à la présence descristaux de nitrates Cette dilatation n'est pas observable sur l'échantilion non twaité en raison du retrait important qui masque le phénomène.

La spectroscopie IR en transmission sur pastille de $\mathrm{KBr}$ a confirmé les résultats obtenus par diffraction des RX. Les bandes correspondant aux vibrations des groupements $\mathrm{NO}_{3}{ }^{-}\left(1360 \mathrm{~cm}^{-1}, 830 \mathrm{~cm}^{-1}\right)$ disparaissent lors de ${ }^{3}$ 'élévation en température du gel.

4. Discussion. La prenière observation que 1 'on peut faire concerne l'élimination des nitrates. Il y a réaction entre la phase amorphe (Sizice) et les phases cristallisées (nitrates) qui donne lieu à l'obtention. d'un matériau amorphe. I'effet de la nature de l'alcalin sur la cristallisation a été mis

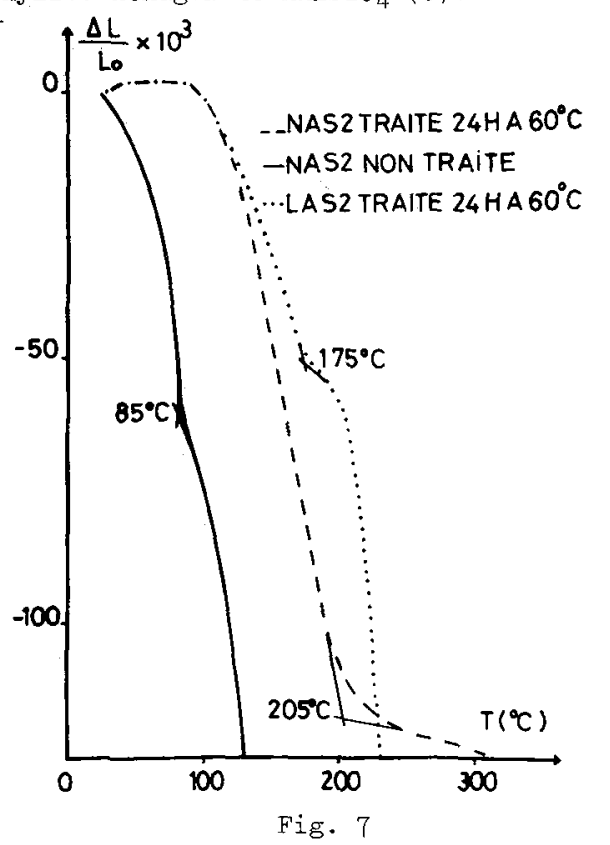


en évidence. Le sodium et surtout le lithium ont f'avorisé 1'apparition des cristaux, comme cela était prévisible (4). Lors de leur cristallisation les gels comme les verres du système $\mathrm{Li}_{2} \mathrm{O}-\mathrm{Al}_{2} \mathrm{O}_{3}-\mathrm{SiO}_{2}$, précipitent du spodumène, cenendant la température de cristallisation est trop basse pour permettre le frittage du matériau. Lorsque l'on passe du lithium au sodium puis au potassium le domaine de température correspondant au gel amorphe augmente, si bien que la réagglomération des grains sera possible pour les gels.KAS. Dans tous les cas les phasesqui sont apparues initialement étaient des phasesternaires. Ce résultat doit être attribué à la présence de l'alumine. Bn effet pour les gels binaires $\mathrm{SiO}_{2}-\mathrm{Na}_{2} \mathrm{O}$ il y a toujours cristallisation sous forme de cristobalite comme phase primaîre de précipitation (5). Les résultats obtenus qui ont été comparés avec ceux de la littérature $(6,7)$ font état du caractére inhibiteur de cristallisation de l'alumine.

La comparaison gel 1 gel 2 est d'un grand intérêt puisque contrairement à ce que l'on pourrait prévoir ( $\zeta$ ) les gels 1 ne cristallisent pas plus tôt que les gelsa; la tendance est même plutôt inversée. Il remarquable qu'un gel KAS 1 ne cristallise pas tandis qu'un gel de silice obtenu par la méthode 1 précipite sous forme de cristobalite aprés un traitement d'une heure seulement aे $950^{\circ} \mathrm{C}$.

5. Conclusion, - L'aptitude à Ia cristallisation des gels $\mathrm{M}_{2} \mathrm{O}-\mathrm{Al}_{2} \mathrm{O}_{3}-6 \mathrm{SiO}_{3}$ où $\mathrm{M}$ est un alcalin, correspond approximativement à celle des verres de même composition. Cependant dans les gels I'apparition des cristaux est beaucoup plus rapide en raison trés certainement de leur grande surface spécifique et de leur importante teneur en eali.

Les gels du système $\mathrm{K}_{2} \mathrm{O}-\mathrm{Al}_{2} \mathrm{O}_{3}-6 \mathrm{SiO}_{2}$ obtenus par la méthode 1 n'ont pas cristallisé aprés une heure de traitement à $1100^{\circ} \mathrm{C}$. Il y a là une possibilité d'utilisation du matériau comme composé de base pour la synthèse de vitrocéramiques ou a'émaux (avec addition d'oxydes adéquats). Dans ce cas on éviterait la fusion préalable, le refroidissement et le broyage du verre. Il suffirait de porter les gels à. la température désirée. D'autre part les compositi ons étudiées sont trés utilisées dans la technologie des céramiques dentaires (9). Les études de synthèse, de limitation des domaines d'obtention et d'volution structurale des gels s'avèrent indispensables pour connaître l'exacte étendue des potentialités de ces matériaux.

\section{Références.}

1. JABRA R., PHALIPPOU J., ZARZYCKI J., J.Non-Crystalline Solids 42 ( 1980$) 489$.

2. SCHOLaE H., Le verre ed. Institut du verre. Paris 1974.

3. PHALIPPOU J., PRASSAS M., ZARZYCKI J., Verres et Refract. 35 (6) (1981) 975.

4. MATUSITA K., TASHIRO M., J.Non-Crystaliine Solids 11 (1973) 471.

5. PRASSAS M., PHALIPPOU J., HENCH I.L, ZARZYCKI J, J. Non Crystaliine Solids $48(1982) 79$

6. PANCRAZI F., PHALIPPOU J., ZARZYCKI J., Ce Congrès.

7. PHALIPPOU J., PRASSAS M., ZARZYCKI J, J. Non Crystalline Solids 48 (1982) 17

8. PHALIPPOU J., ZARZYCKI J., LALANNE J.F., Ann.Chim.Fr. 3 (1978) 99.

9. HAHN C., TEUCHERT K., Ber.Dt.Keram. ges. $27(9 / 10)(1980) 208$. 